\title{
Phencyclidine Exacerbates Attentional Deficits in a Neurodevelopmental Rat Model of Schizophrenia
}

\author{
Gwenaëlle Le Pen*,', Andrew J Grottick², Guy A Higgins ${ }^{3}$, Jean-Luc Moreau ${ }^{3}$ \\ 'INSERM-EMI O1 17, Centre Paul Broca, Paris, France; ${ }^{2}$ Arena Pharmaceuticals Inc., San Diego, CA, USA; ${ }^{3}$ Pharma Division, Preclinical CNS \\ Research, F-Hoffmann La Roche Ltd, Basel, Switzerland
}

\begin{abstract}
Schizophrenia is characterized by severe abnormalities in cognition, including disordered attention. In the rat, neonatal ventral hippocampal (NVH) lesions induce behavioral abnormalities at adulthood thought to simulate some aspects of the symptomatology of schizophrenia. Here, we compared the effects of $\mathrm{NVH}$ and adult ventral hippocampal (AVH) lesions on attentional performance as assessed by the five-choice serial reaction time task (5-CSRTT). NVH-lesioned rats were slower to acquire the task than AVH-lesioned and control animals. When training was complete, $\mathrm{NVH}$ - and $\mathrm{AVH}$-lesioned animals exhibited stable but disrupted performance under standard conditions, thus emphasizing an implication of $\mathrm{VH}$ in visual attentional processes. Variations in task parameters induced a significantly greater disruption in $\mathrm{NVH}$ - and $\mathrm{AVH}$-lesioned groups as compared to controls. $\mathrm{NVH}$-lesioned rats were also hyperresponsive to the disruptive effects of a high dose of phencyclidine (PCP) $(3 \mathrm{mg} / \mathrm{kg})$. In contrast, amphetamine $(0.4-0.8 \mathrm{mg} / \mathrm{kg})$ had a similar effect in control and $\mathrm{VH}$-lesioned rats. Thus, $\mathrm{NVH}$-lesioned rats were impaired in the acquisition of stable performance in the 5-CSRTT, and were hypersensitive to the cognitive-impairing effects of PCP.

Neuropsychopharmacology (2003) 28, 1799-1809, advance online publication, 4 June 2003; doi: I 0. I 038/sj.npp. 1300208
\end{abstract}

Keywords: Schizophrenia; animal model; neonatal lesion; ventral hippocampus; attentional processes; five-choice serial reaction time task

\section{INTRODUCTION}

Neuropathological changes in the hippocampus have been frequently observed in schizophrenia (Zaidel et al, 1997; Weinberger, 1999; Csernansky et al, 2002). These brain abnormalities have been proposed to result from abnormal neurodevelopmental processes (Weinberger, 1986). An animal model simulating neurodevelopmental lesions of the hippocampus has been developed by Lipska and coworkers (Lipska et al, 1993; Lipska and Weinberger, 1993). This model, based on the long-term effects of postnatal day7 lesions of the ventral hippocampus, provides preclinical evidence for the neurodevelopmental hypothesis of schizophrenia. Neonatal ventral hippocampal (NVH) lesions in rats have been shown to induce postpubertal emergence of behavioral abnormalities thought to simulate some aspects of both the positive and negative symptomatology of schizophrenia. These include hyper-responsiveness to stress, novelty, dopamine agonists and glutamate antagonists (Lipska et al, 1995a, 1993; Flores et al, 1996; Black et al,

\footnotetext{
*Correspondence: Dr G Le Pen, INSERM-EMI 0I I7, Physiopathologie des maladies psychiatriques, Développement et Vulnérabilité, Centre Paul Broca, 2ter rue d'Alésia, 75014 Paris, France, Tel: +33 |40788635, Fax: + 33 |40788628, E-mail: lepen@broca.inserm.fr Received 20 November 2002; revised 18 March 2003; accepted 21 March 2003

Online publication: 4 April 2003 at http://www.acnp.org/citations/ Npp040402421/default.pdf
}

1998; Brake et al, 1999; Al-Amin et al, 2000, 2001). NVHlesioned rats also exhibit deficits in social behavior (SamsDodd et al, 1997; Becker et al, 1999) and altered reward sensitivity (Le Pen et al, 2002).

Traditionally, schizophrenia has been viewed as an illness comprised primarily of positive and negative symptoms, but it is now recognized that cognitive impairments are also a central feature of this disorder, and not secondary to drug effects or other symptoms (Breier, 1999; Weinberger and Gallhofer, 1997). Cognitive deficits are common across all subtypes of schizophrenia and include deficits in abstraction, executive function, verbal memory, language function, vigilance, and attention (Sharma, 1999; Joober et al, 2002; Hughes et al, 2003).

Regarding cognitive processes, NVH-lesioned rats exhibit a postpubertal emergence of deficits in sensorimotor gating (Lipska et al, 1995b; Le Pen et al, 2000), in latent inhibition (Grecksch et al, 1999), and also in spatial learning and working memory (Chambers et al, 1996; Lipska et al, 2002). More recently, we extended these results by demonstrating lesion-induced deficits in spatial and associative learning (Le Pen et al, 2000).

To further explore the neurodevelopmental aspects of this model and its validity, we investigated the effects of neonatal and adult $\mathrm{VH}$ lesions on attentional processes. As attention subsumes several distinct underlying processes (Robbins and Everitt, 1995; Sarter and Bruno, 2000) including sustained attention, selective attention, and 
executive control, we have used the five-choice serial reaction time task (5-CSRTT) in rats. This test provides independent measures of these different processes in rats (Carli et al, 1983) and exhibits closed analogies with the continuous performance test and Leonard's 5-CSRTT used in humans to assess attentional processes (Mirsky and Rosvold, 1960; Leonard, 1959, 1961). In the 5-CSRTT, rats must detect and respond to a series of brief visual stimuli that are presented in one of five locations in a spatial array. Attentional demands of the task can be increased by reducing brightness or duration of the stimulus, decreasing the time between stimulus presentations, or interpolating unpredictable white noise. Thus, we have assessed and compared the performance of neonatal and adult $\mathrm{VH}$ lesioned rats in the 5-CSRTT under normal conditions and after increasing attentional demands. Finally, the effects of two classic psychotomimetic drugs (phencyclidine (PCP) and amphetamine) on rats' performances have been evaluated.

\section{METHODS}

All animal experiments were approved by local animal protection authorities

\section{Animals}

Sprague-Dawley rat pups were obtained at 3-4 days of age as whole litters together with their mother and adult male Sprague-Dawley rats were obtained at 50 days of age (BRL, Füllinsdorf, Switzerland). After weaning, rats were housed in groups of four in holding rooms at controlled temperature $\left(20-22^{\circ} \mathrm{C}\right)$ with a 12 -h light/12-h dark cycle (ON: $06.00 \mathrm{~h}$; OFF: $18.00 \mathrm{~h}$ ). Behavioral testing occurred during light cycles between 10.00 and $16.00 \mathrm{~h}$.

\section{Surgical Procedure}

Neonatal rats. On the seventh day of age and at a body weight of $15-20 \mathrm{~g}$, male pups within each litter were randomized to Sham or Lesion status, anesthetized by isoflurane inhalation ( $4 \%$ for induction and $1.5-3 \%$ for maintenance) through a mask, mounted on a stereotaxic Kopf instrument with an adapter for small animals (Harvard Biosciences) and additionally taped on a heating pad placed on the platform. The skin overlying the skull was incised and $0.3 \mu \mathrm{l}$ of either ibotenic acid (Sigma, St Louis, MO, USA; $10 \mu \mathrm{g} / \mu \mathrm{l}$ ) or artificial cerebrospinal fluid was bilaterally infused over a 2-min period by a micro-infusion pump (PHD Programmable, Harvard Biosciences) using an injection cannula $(0.3 \mathrm{~mm}$ diameter $)$ aimed at the ventral hippocampal formation (AP $-3.0 \mathrm{~mm}, \mathrm{ML} \pm 3.5 \mathrm{~mm}$, and $\mathrm{DV}-5.0 \mathrm{~mm}$ relative to bregma). After completion of the infusion, the cannula was left in place for an additional 3min period before being slowly removed. Then, the skin overlying the skull was sutured and the animals were allowed to recover on a heating pad before being returned to their mother (female pups were removed from the litter). Eighteen days after surgery (ie PND25) rats were weaned and housed four per cage until further testing.

Adult rats. We anesthetized 56-day-old rats with ketamine $80 \mathrm{mg} / \mathrm{kg}$ (Ketalar, Parke-Davis) and xylazine $10 \mathrm{mg} / \mathrm{kg}$
(Rompun, Bayer). The animals were mounted on a stereotaxic Kopf instrument and the skin overlying the skull was incised. Then, $2 \times 0.2 \mu \mathrm{l}$ of either ibotenic acid (Sigma, St Louis, MO, USA; $15 \mu \mathrm{g} / \mu \mathrm{l}$ ) or artificial cerebrospinal fluid was bilaterally infused over a 2-min period by a micro-infusion pump (PHD Programmable, Harvard Biosciences) using an injection cannula $(0.3 \mathrm{~mm}$ diameter $)$ aimed at the ventral hippocampal formation (AP $-4.8 \mathrm{~mm}$, $\mathrm{ML} \pm 5.2 \mathrm{~mm}$, and $\mathrm{DV}-7.0$ and $-5.0 \mathrm{~mm}$ relative to bregma). After completion of the infusion, the cannula was left in place for an additional 3-min period before being slowly removed. This procedure was adapted from Lipska et al (1993) in order to induce lesions similar in volume and location to those obtained after neonatal injections of ibotenic acid. Following infusion, the skin overlying the skull was sutured and the animals were allowed to recover on a heating pad before being returned to their home cage.

\section{Five-Choice Serial Reaction Time Task}

For the 5-CSRRT, animals were maintained under a schedule of restricted access to food ( $1 \mathrm{~h}$ a day after experimentation) in order to maintain $85 \%$ of free feeding body weight. Except during testing, water was always available ad libitum.

Apparatus. Five-choice operant chambers (Med Associates Inc., St Albans, VT) housed in sound insulated and ventilated enclosures were used for all experiments. Each chamber consisted of an aluminum enclosure $(25 \times 30 \mathrm{~cm})$ containing on one wall a food hopper and house light and on the opposite wall an array of five square holes $(2.5 \times 2.5 \times 2.5 \mathrm{~cm})$ arranged on a curved panel and raised $2.5 \mathrm{~cm}$ from the grid floor. A LED (standard conditions: $150 \mathrm{~lx}$ ) was positioned at the rear of each hole. All apertures in the chamber, including the food hopper, were controlled by a photocell placed across the entrance. Operant chambers were controlled by the Kestrel Control System (Conclusive Solutions, Harlow, UK).

Training. Training began with the illumination of the house light and delivery of a food pellet. A nose-poke into the magazine tray started the first trial, which consisted of an intertrial interval (ITI, $5 \mathrm{~s}$ ) followed by the random illumination of one of the five lights for a fixed interval (stimulus duration, SD). If a nose-poke was registered in the illuminated niche before the end of either the SD, or a fixed interval after this period (limited hold, LH) a further pellet was dispensed and a 'correct trial' registered. An incorrect nose-poke (incorrect trial) or failure to respond within the allotted time (missed trial) resulted in a time-out (TO) period during which the house light was extinguished for $5 \mathrm{~s}$. Responding to one of the five niches during the ITI (premature response), or after a correct trial was registered (perseverative response), resulted in a further TO. Finally, if a rat responded into a niche during a TO, the TO was restarted. Each training session ran for either 100 trials or $60 \mathrm{~min}$, whichever was shorter.

Task acquisition. Rats were initially given access to a handful of pellets ( $45 \mathrm{mg}$ Noyes Formula P Food Pellets) in 
their home cage for 2 consecutive days. Training commenced with daily 60 -min sessions, in which subjects were placed in the operant chambers and both the food hopper and five light niches were filled with approximately five pellets each. On subsequent days, no food was placed in the chambers before sessions began.

Initially, stimulus parameters were such that $\mathrm{SD}$ was set at $30 \mathrm{~s}$ and ITI, TO and LH were $5 \mathrm{~s}$. Training continued until the rats achieved $80 \%$ correct responses ([correct/(correct + incorrect) $] * 100$ ) and less than $20 \%$ omissions on 2 consecutive days. Then, SD was progressively reduced to $15 \mathrm{~s}$. After 2 consecutive days of consistent performances at this criterion, SD was further reduced to $5 \mathrm{~s}$, then $2 \mathrm{~s}$. To finally reach $1 \mathrm{~s}$, rats must have reached the target criterion for 3 consecutive days. At $1 \mathrm{~s}$ SD, to avoid overtraining, rats with good performance were only tested twice a week.

To compare acquisition of this task between the different groups of rats, the number of days to reach the next level of SD was evaluated. Arbitrarily, when SD was 30 s, day 1 was considered when 10 correct trials had been registered for a rat.

After the exclusion of rats following histological examination, we have noticed that five NVH-lesioned rats never reached the $2 \mathrm{~s} S \mathrm{SD}$ criterion even after a large number of training sessions. Moreover, at $1 \mathrm{~s} \mathrm{SD}$, some rats (one in the adult lesion group, and three in the neonatal sham group) completed only $60 \%$ or less of the total number of trials indicating very low performances. A Pearson $\chi^{2}$ test revealed no significant effect of lesion on the proportion of rats that failed to acquire the task in both adult and neonatal groups $\left(\chi^{2}=0.87\right.$, NS and $\chi^{2}=0.96$, NS; respectively). In addition, a Breslow and Day (BD) test for homogeneity of odds ratios indicated that the profiles obtained in adult or neonatal operated animals were similar (BD coefficient $=0.37, \mathrm{NS}$ ). Therefore, to compare groups with homogeneous performances, rats that failed to acquire the task properly were excluded from the analysis and presentation.

Acquisition data and data under standard conditions were analyzed using a three-factor ANOVA (lesion status $\times$ age at lesion $\times$ training stage or day, respectively) with repeated measurements on factors training stage or day respectively, followed when appropriate by separate two-factor ANOVA or by the Fisher's PLSD post hoc test.

Task manipulation. Training continued until subjects had achieved stable performances (more than $80 \%$ correct responses and less than $20 \%$ omissions) for at least a 2-week period.

To assess the effects of changing the stimulus parameters, rats were exposed to (1) standard or reduced stimulus intensity (SI), respectively, in arbitrary units $(\mathrm{SI}=255$ : normal intensity; $\mathrm{SI}=1$ : low intensity), each presented randomly 50 times during the test session, (2) standard or reduced stimulus duration $(\mathrm{SD}=1$ or $0.05 \mathrm{~s}$, respectively, with equal numbers of each SD randomly presented during the session), (3) a distracting white noise $(85 \mathrm{~dB}, 0.5 \mathrm{~s}$ duration) or no noise presented at the end of the ITI (simultaneously with the visual target stimulus) in a random and counterbalanced procedure, $(4)$ standard (5s) or longer (10s) ITI duration, each presented randomly 50 times during the session.

In all cases rats were given 100 trials and $60 \mathrm{~min}$ to complete the session (except for the session on ITI duration, which lasted more than $60 \mathrm{~min}$ ). Task manipulation occurred on Tuesdays and Fridays. For the rest of the week all subjects were run under standard conditions.

Data were analyzed using a three-factor ANOVA (lesion status $\times$ age at lesion $\times$ stimulus parameter) with stimulus parameter as a repeated measure factor followed when appropriate by separate two-factor ANOVA or by the Fisher's PLSD post hoc test.

Psychotomimetic challenges. Following manipulation of stimulus parameters, baseline performance was re-established for a week. The effects of two classic psychotomimetic drugs (amphetamine, a dopamine agonist, and PCP, a glutamate receptor antagonist) were evaluated using standard stimulus parameters $(\mathrm{SD}=1 \mathrm{~s}$; ITI, TO, and $\mathrm{LH}=5 \mathrm{~s})$.

D-amphetamine and PCP were dissolved in $0.9 \%$ saline and administered in a volume of $5 \mathrm{ml} / \mathrm{kg}$ body weight, 10 min prior to the start of the test session. Drug challenges occurred on Tuesdays and Fridays. The other days of the week were devoted to training sessions under standard conditions. First animals received PCP $(0,0.3,1$, or $3 \mathrm{mg} / \mathrm{kg}$, s.c.) and then amphetamine $(0,0.4$, or $0.8 \mathrm{mg} / \mathrm{kg}$, i.p.) according to a Latin Square design. Rats were kept in training under baseline conditions for a week between the PCP and the amphetamine experiment. Doses of amphetamine and PCP used in the present experiments were selected from previous reports in the literature (eg Cole and Robbins, 1987; Harrison et al, 1997; Jin et al, 1997).

Data were analyzed using a three-factor ANOVA (lesion status $\times$ age at lesion $\times$ drug dose) with repeated measurements on factor drug dose, followed when appropriate by separate two-factor ANOVA or by the Fisher's PLSD post hoc test.

\section{Locomotor Activity}

Locomotor activity was assessed in a Digiscan actimeter (Omnitech Electronic Inc., Colombus, $\mathrm{OH}$ ) monitoring horizontal and vertical movements of the animals. The individual compartments $(40 \times 40 \times 30 \mathrm{~cm})$ were located in a dimly lit and quiet room. Horizontal and vertical activity counts were recorded for a 60-min period, and were expressed as the total number of horizontal and vertical beams crossed by the animal. Locomotor activity data were analyzed using a two-factor ANOVA (lesion status $\times$ age at lesion), followed when appropriate by separate two-factor ANOVA or by the Fisher's PLSD post hoc test.

\section{Rating of Lesion Size (according to Sams-Dodd et al, 1997)}

At the completion of behavioral evaluation, rats were killed by decapitation. Brains were rapidly removed and after fixation in formalin solution $(10 \%$ in $\mathrm{NaCl}$ for at least 1 week), $40-\mu \mathrm{m}$ sections were sliced with a freezing cryostat. The sections through the lesioned area were mounted and stained with cresyl violet. The extent of the lesion on each 
side of the brain was rated as follows: 0 - no discernible cell loss in the hippocampal formation, 1-small, 2-medium, 3 - large area of cell loss within the ventral hippocampal formation. Scores for both sides were added to yield a total score ranging from 0 to 6 .

\section{RESULTS}

\section{Histology}

One NVH-lesioned rat was excluded because of extrahippocampal damage (cortex) and another one because of unilateral lesion. Thus, following histological examination and removal of animals that did not acquire the 5-CSRTT properly, 7 and 10 control and 9 and 11 lesioned rats were included for data analysis in the neonatal-lesioned and the adult-lesioned group, respectively. It has to be noted that rats excluded from the analysis because of their poor learning performances exhibited lesion scores similar to those obtained for rats included in the analysis.

Neonatal- and adult-lesioned rats exhibited mean lesion scores of $3.5 \pm 0.4$ and $3.4 \pm 0.2$, respectively, indicating (1) a similar extent and location of the lesion in adult- and neonatal-lesioned animals (Figure 1), and (2) that the cell loss was restricted to the ventral part of the hippocampus. Some animals exhibited cavitation around the site of injection. In control rats injected with artificial cerebrospinal fluid, the hippocampus was morphologically intact (lesion score 0 ).

a

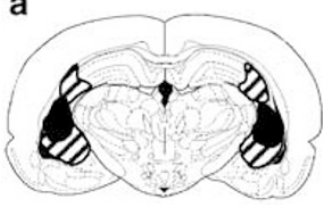

Bregma $-4.3 \mathrm{~mm}$

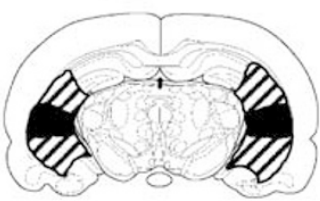

Bregma $-5.3 \mathrm{~mm}$

b

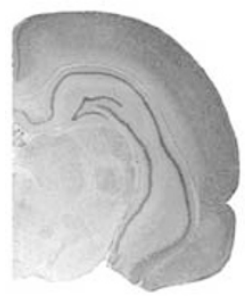

C

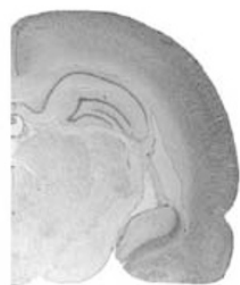

d

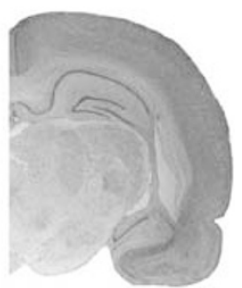

Figure I Lesion boundaries in the ventral hippocampus of rats infused bilaterally with ibotenic acid. (a) Schematic drawing of the ventral hippocampus with boundaries of the largest (stripes) and smallest (black) lesions. Representative photomicrographs of cresyl violet-stained coronal section through the brains of rats that had received sham (b), neonatal (c), or adult (d) lesions of the ventral hippocampus.

\section{Five-Choice Serial Reaction Time Task}

Task acquisition. The rate of acquisition of the 5-CSRTT by neonatal- and adult-lesioned animals is illustrated in Figure 2. A three-way ANOVA revealed a significant effect of age at lesion $(\mathrm{F}(1,33)=14.3, p<0.001)$, a significant effect of lesion $(\mathrm{F}(1,33)=22.7, p<0.001)$, and a significant effect of training stage $(F(3,99)=23.2, p<0.001)$. There was also a significant age $\times$ lesion interaction $(F(1,33)=12.3$, $p<0.01$ ), indicating that neonatal and adult lesions differentially affected performance at the various stages of training. Post hoc comparisons revealed that, in contrast to AVH-lesioned or control animals, NVH-lesioned rats needed more time to reach a consistent performance criterion at each stage of the training $(30 \mathrm{~s}, p<0.01 ; 15 \mathrm{~s}$, $p<0.01 ; 5 \mathrm{~s}, p<0.05 ; 2 \mathrm{~s}, p<0.01)$.

Performance under standard conditions. Figure 3 shows the mean performance of adult- and neonatal-lesioned rats in the 5-CSRTT over a 10-day period under standard conditions. Rats were tested once daily for 10 days when stage SD $1 \mathrm{~s}$ was reached. Both control and VH-lesioned rats were able to acquire stable responses as revealed by no significant day effects and no significant interactions with factor day upon percent correct and correct latency. However, VH lesions significantly decreased accuracy (percent correct responses) $(\mathrm{F}(1,33)=14.9, p<0.001)$, increased correct latency $(\mathrm{F}(1,33)=7.9, p<0.01)$, and also largely tended to increase the number of perseverative responses $(\mathrm{F}(1,33)=3.98, p=0.054)$. These effects occurred independently of lesion age as indicated by a lack of significant age or age $\times$ lesion interactions across any of the parameters studied.

\section{Task manipulation}

Increased ITI: The effect of increasing ITI on accuracy and correct latency is illustrated in Figure $4 \mathrm{a}$ and $\mathrm{b}$. Accuracy was significantly decreased by the lesion $(\mathrm{F}(1,33)=5.3, p<0.05)$ and by the increase of ITI

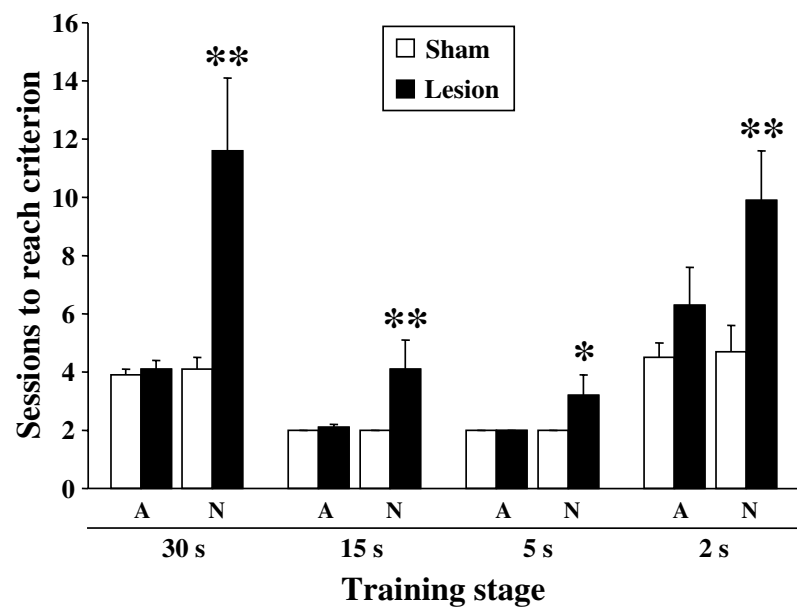

Figure 2 Effect of neonatal $(N)$ or adult $(A)$ ventral hippocampal lesions on acquisition of the 5-CSRTT. The success criterion is defined as the number of days to reach $80 \%$ correct responses with $<20 \%$ omissions with a SD of $30,15,5$, and $2 \mathrm{~s}$. ${ }^{*} p<0.05$ and $* * 00.01$ compared to respective sham animals. 

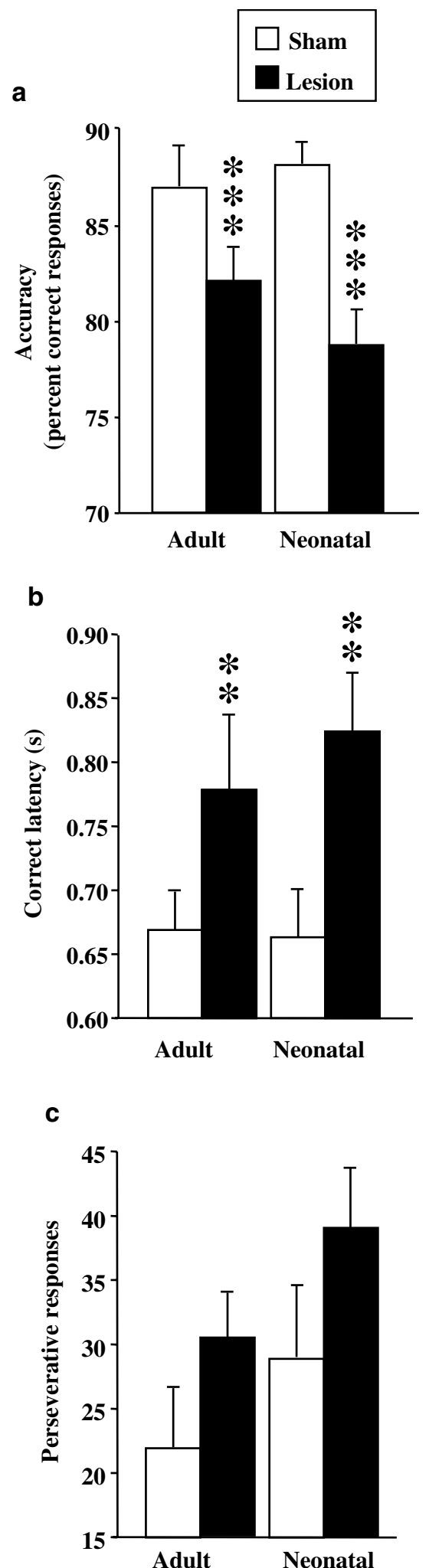

Figure 3 Effect of ventral hippocampal lesions performed neonatally or at adulthood on mean performance over 10 consecutive days of testing under standard test conditions in the 5-CSRTT. Data shown are for percentage correct responses (a), correct latency (b), and perseverative responses (c). $* * * 0.01$ and ${ }^{*} * * * 0.001$ compared to controls.

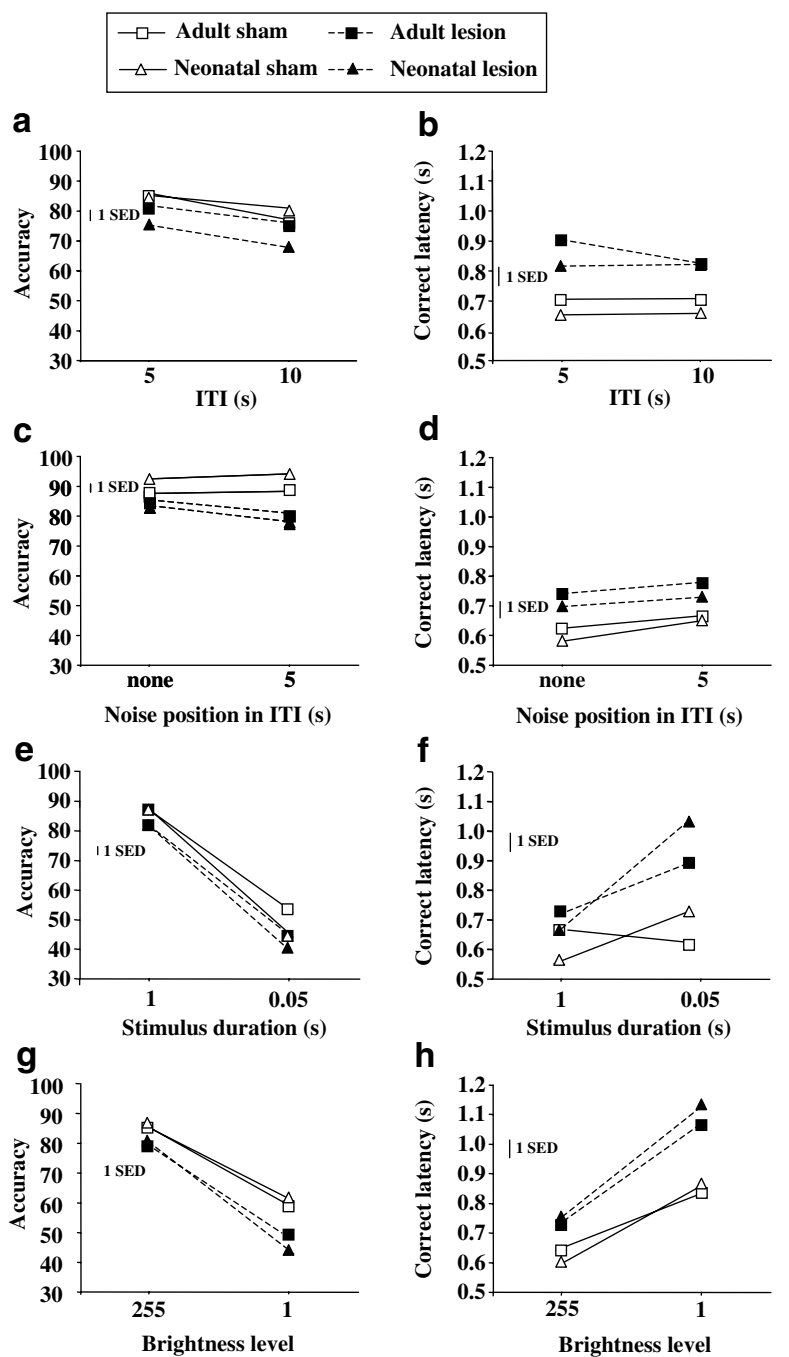

Figure 4 Effect of neonatal and AVH lesions on performances (percent correct responses and correct latency) under various task manipulations, such as variable ITI (a, b), white noise distractor (c, d), variable SD (e, f), and variable $\mathrm{SI}(\mathrm{g}, \mathrm{h})$.

$(\mathrm{F}(1,33)=11.7, p<0.01)$. No significant two- or three-way interactions were noted, indicating a similar effect of increasing ITI on both sham and VH-lesioned animals whatever the age of lesion. An increase of correct latency was also observed after both neonatal and adult $\mathrm{VH}$ lesion $(\mathrm{F}(1,33)=5.3, p<0.05)$ but no significant two- or three-way interactions were found. Thus, increasing ITI had no significant effect on the increase of correct latency induced by the lesion in both adult- and neonatal-lesioned rats.

Interpolation of a white noise distractor: The effects of interpolating a white noise distractor on accuracy and correct latency are illustrated in Figure $4 \mathrm{c}$ and d. Here again, accuracy was decreased by the lesion $(\mathrm{F}(1,33)=10.4$, $p<0.01)$ but no significant effect of age, or age $\times$ lesion interaction $(F(1,33)=0.3$, NS and $F(1,33)=2$, NS, respectively). A significant noise distractor $\times$ lesion interaction $(\mathrm{F}(1,33)=7.9, p<0.01)$ indicated that the decrease in accuracy induced by the noise distractor was only observed in VH-lesioned rats. An increase of correct latency was also 
observed in all groups after interpolation of a noise distractor $(\mathrm{F}(1,33)=7.9, p<0.01)$.

Reduced SD: The effect of reducing SD on accuracy and correct latency is illustrated in Figure $4 \mathrm{e}$ and $\mathrm{f}$. Accuracy was significantly decreased by the lesion $(\mathrm{F}(1,33)=8.3$, $p<0.01)$ and by the decrease of $\mathrm{SD}(\mathrm{F}(1,33)=503$, $p<0.001)$. No significant two- or three-way interactions were noted, indicating a similar effect of decreasing SD in both sham and $\mathrm{VH}$-lesioned animals, irrespective of age at lesion. An increase of correct latency was also observed after $\mathrm{VH}$ lesions $(\mathrm{F}(1,33)=9.7, p<0.01)$ and following the decrease of $\operatorname{SD}(\mathrm{F}(1,33)=20.9, p<0.001)$. A significant $\mathrm{SD} \times$ lesion interaction was found $(\mathrm{F}(1,33)=8, p<0.01)$ indicating that the increase in correct latency induced by the reduction of SD was more pronounced in $\mathrm{VH}$-lesioned rats, again, regardless of age at lesion.

Reduced brightness level: Figure $4 \mathrm{~g}$ and $\mathrm{h}$ shows the effects of reducing SI on accuracy and correct latency. Accuracy was significantly decreased by the lesion $(\mathrm{F}(1,33)=9.7, \quad p<0.01)$ and by the reduction of SI $(\mathrm{F}(1,33)=400, p<0.001)$. A significant $\mathrm{SI} \times$ lesion was observed $(\mathrm{F}(1,33)=6.5, p<0.05)$ but no other two- or three-way interactions were found, indicating that the decrease of accuracy induced by the reduction of SI was more pronounced in $\mathrm{VH}$-lesioned rats than in controls whatever the age of lesion. An increase of correct latency was also observed after lesioning $(\mathrm{F}(1,33)=10.2, p<0.01)$ and after decreasing SI $(\mathrm{F}(1,33)=67, p<0.001)$. No other significant two- or three-way interactions were found, indicating that the decrease of correct latency induced by the reduction of SI was more pronounced in $\mathrm{VH}$-lesioned rats than in controls whatever the age of lesion.

Effect of psychotomimetics upon performance in the 5CSRTT. The effects of amphetamine and PCP on 5-CSRTT under standard conditions are depicted in Figure $5 \mathrm{a}$ and $\mathrm{b}$, respectively.

Amphetamine: Accuracy was significantly decreased by the lesion $(\mathrm{F}(1,33)=7, p<0.05)$ and by amphetamine $(\mathrm{F}(2,66)=14.3, p<0.001)$. No two- or three-way interactions were observed, indicating that the decrease of accuracy induced by amphetamine was similar in control and $\mathrm{VH}$-lesioned rats whatever the age of lesion.

Amphetamine also decreased correct latency $(\mathrm{F}(2,66)=$ 23.7, $p<0.001)$, the number of correct trials $(\mathrm{F}(2,66)=14.7$, $p<0.001)$, and increased premature and perseverative responses $(\mathrm{F}(2,66)=15, p<0.001$ and $\mathrm{F}(2,66)=31.6$, $p<0.001$, respectively) (data not shown). All these modifications reached significant levels for $0.8 \mathrm{mg} / \mathrm{kg}$ of amphetamine except for correct latency, which was also significantly increased at $0.4 \mathrm{mg} / \mathrm{kg}$. In addition, number of omission and magazine latency were not modified by amphetamine $(\mathrm{F}(2,66)=1.9$, NS and $\mathrm{F}(2,66)=0.5$, NS, respectively). No two- or three-way interactions were observed, indicating that these modifications induced by amphetamine were similar in control and lesioned rats whatever the age of lesion.

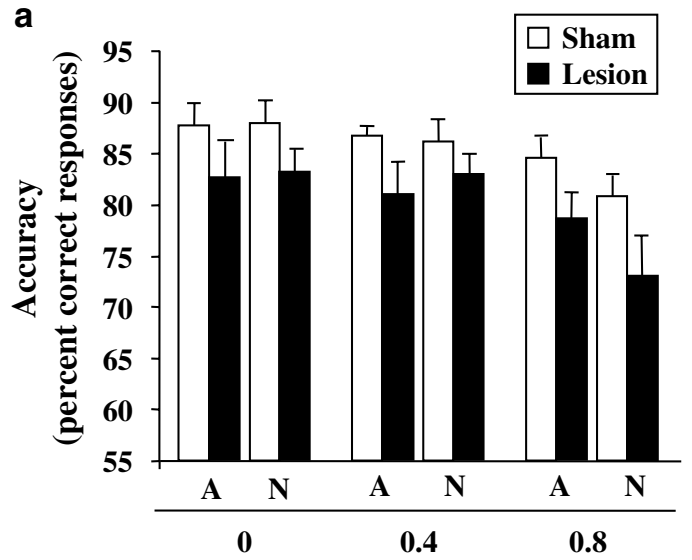

Amphetamine dose (mg/kg)

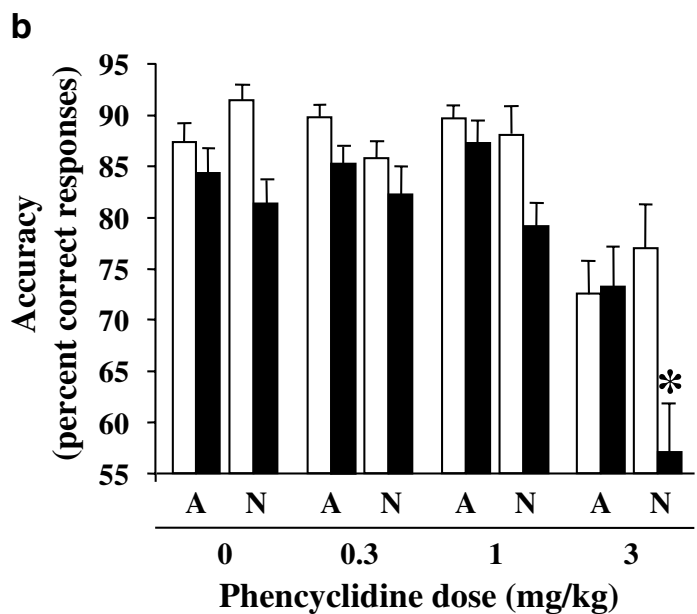

Figure 5 Effect of amphetamine (a) and PCP (b) on performances in the 5-CSRTT in rats with neonatal $(N)$ or adult $(A)$ ventral hippocampal lesions. ${ }^{*} p<0.05$ in comparison with respective controls.

Phencyclidine: We show a significant decrease of accuracy with lesion $(\mathrm{F}(1,33)=13.1, p<0.001)$ and with PCP dose $(F(3,99)=36.8, p<0.001)$. A significant age $\times$ lesion interaction was observed $(\mathrm{F}(1,33)=5.4, p<0.05)$, indicating that $\mathrm{NVH}$-lesioned rats were more sensitive to the disruptive effect of PCP compared to sham and adult VH-lesioned rats. A significant lesion and age $\times$ lesion $\times$ PCP interaction $(\mathrm{F}(1,33)=13.1, p<0.001$ and $\mathrm{F}(3,99)=2.9, p<0.05$ respectively) followed by separate ANOVA for each PCP dose revealed that hypersensitivity to PCP observed in NVH-lesioned rats was only seen at $3 \mathrm{mg} /$ $\mathrm{kg}$ (significant age $\times$ lesion interaction: $\mathrm{F}(1,33)=5.8$, $p<0.05)$.

PCP also induced an increase of correct latency, missed trials, premature and perseverative responses $(\mathrm{F}(3,99)=$ 28.5, $p<0.001, \quad \mathrm{~F}(3,99)=47.7, \quad p<0.001, \quad \mathrm{~F}(3,99)=4.8$, $p<0.01$, and $\mathrm{F}(3,99)=7, p<0.001$, respectively), and a decrease in the number of correct trials $(F(3,99)=60$, $p<0.001)$ (data not shown). All these modifications reached significant levels for $3 \mathrm{mg} / \mathrm{kg}$ of PCP except for premature and perseverative responses, which were also significantly increased at $1 \mathrm{mg} / \mathrm{kg}$. No two- or three-way interactions were observed, indicating that these modifications induced 


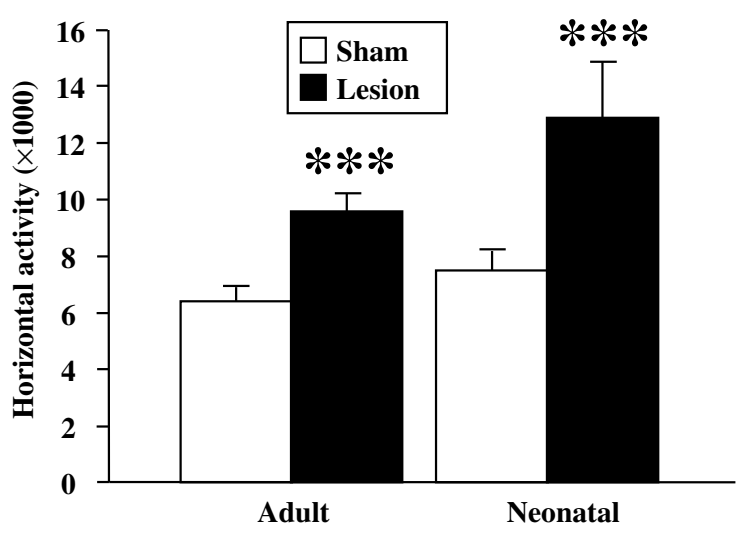

b

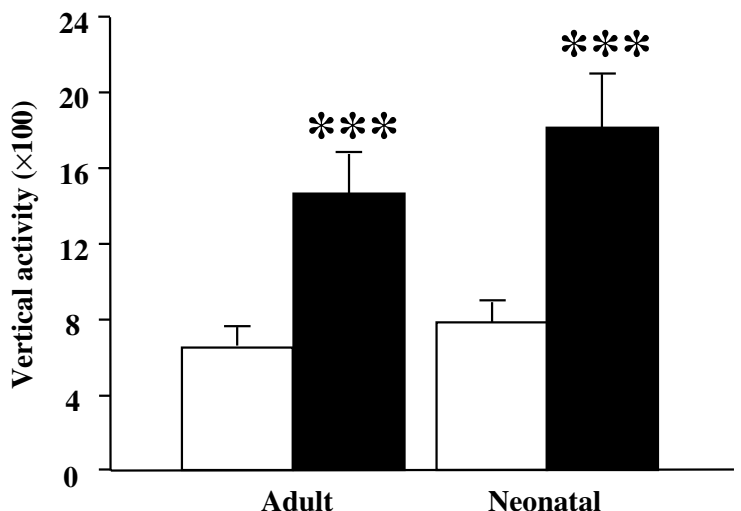

Figure 6 Effect of neonatal or AVH lesions on spontaneous horizontal (a) and vertical (b) activity within an open field chamber over a 60-min period. $*^{*} * *<0.001$ in comparison with controls.

by PCP were similar in control and lesioned rats whatever the age of lesion.

\section{Locomotor Activity}

Spontaneous horizontal (Figure 6a) and vertical (Figure 6b) activity was significantly increased in lesioned rats as compared to sham-operated animals $(\mathrm{F}(1,33)=13.7$, $p<0.001$ and $\mathrm{F}(1,33)=19.7, p<0.001$, respectively). No significant effect of age or age $\times$ lesion interaction was observed for the two parameters studied, indicating that the increase of both horizontal and vertical spontaneous activity induced by the lesion was similar whatever the age at lesion.

\section{DISCUSSION}

In the present study, we investigated the effects of neonatal and AVH lesions on attentional processes as assessed by the 5-CSRTT. We compared control and lesioned rats for their task acquisition, baseline performance, and for their response to various parametric manipulations or psychotomimetic drugs administration.

\section{Task Acquisition}

At long SDs (training stage 30,15, and $5 \mathrm{~s}$ ), control, adult $\mathrm{VH}-$ lesioned and neonatal VH-lesioned rats were successfully trained in the 5-CSRTT. However, NVH-lesioned rats required more trials than control or AVH-lesioned subjects to reach the criterion at each training stage. Thus, $\mathrm{NVH}-$ lesioned rats exhibited a clear deficit in the initial acquisition of the stimulus light-reward association. This acquisition deficit could result from a difference in exploratory drive between neonatal and AVH-lesioned rats. This is unlikely since both adult- and neonatal-lesioned rats exhibited similar increases in locomotor activity in a novel open-field. This deficit could also be explained by the fact that NVH-lesioned rats may be less motivated to learn the task. Indeed, latency to collect the earned food pellets, a sensitive index of the level of primary motivation (Carli and Samanin, 1992; Harrison et al, 1997), was slightly higher in $\mathrm{NVH}-$ lesioned rats than in AVH-lesioned and control rats. Moreover, we have recently shown that, in contrast to adultlesioned rats, neonatal-lesioned animals exhibited deficits in reward sensitivity (Le Pen et al, 2002). In addition, NVHlesioned rats are also impaired in their capacity to acquire and retain information in tests of spatial and avoidance learning (Le Pen et al, 2000) whereas AVH lesions have a smaller impact on these procedures (Moser et al, 1995; Stubley-Weatherly et al, 1996; Hock and Bunsey, 1998). Thus, the slower acquisition of the task in NVH-lesioned rats may be best explained by reward and mnemonic process deficits, both of which are required to learn the initial association between stimulus presentation, operant response, and reward.

Offspring of mothers that show poor levels of pup licking and grooming also exhibit poor spatial learning and locomotor performances (Liu et al, 2000; Gomez-Serrano et al, 2001). According to these results, cognitive deficits seen in NVH-lesioned rats during the acquisition phase could result from reduced maternal care. However, a recent study of the behavioral outcome of NVH lesions in two strains of rats exhibiting differences in the frequency of maternal behavior has revealed no difference of maternal care towards lesioned or control pups (Wood et al, 2001). Nevertheless, NVH-lesioned rats were significantly more affected by increases in arched-back nursing compared to controls (Wood et al, 2001). Thus, increased maternal interactions perceived to be beneficial in some models could actually lead to some of the deficits seen in NVH-lesioned rats.

In the second part of the training, when $\mathrm{SD}$ was reduced to $2 \mathrm{~s}$, several $\mathrm{NVH}$-lesioned rats failed to reach the criterion. Moreover, NVH-lesioned rats that reached the criterion required significantly more sessions to do so than controls and rats lesioned at adulthood. In addition, AVHlesioned rats also tended to need more sessions than controls to reach criteria. Altogether, these deficits already reflect an effect of $\mathrm{VH}$ lesions on attentional processes.

\section{Effects of Lesions upon Baseline Performance in the 5-CSRTT}

When stable performances had been reached at $1 \mathrm{~s} S \mathrm{SD}$, subjects with neonatal and adult $\mathrm{VH}$ lesions were less likely, 
and slower to make a correct response as compared to controls. An almost identical pattern of deficits can be observed after lesions of the basal forebrain cholinergic systems, which are known to be crucial for sustained and executive control of performance (Robbins and Everitt, 1995; Sarter and Bruno, 2000). More interestingly, adult and neonatal VH lesion-induced deficits in accuracy were smaller than those found after lesions of the entire medial prefrontal cortex (mPFC) and more similarly to those obtained after lesions of the dorsal or ventral portions of the mPFC (cingulate and prelimbic-infralimbic (PRL-IL) areas, respectively) (Muir et al, 1994, 1996; Passetti et al, 2002). Similarly to PRL-IL lesions, these deficits resulted from an increase in incorrect responses. However, in contrast to PRL-IL lesions, $\mathrm{VH}$ lesions increased the latency to respond correctly to a lower extent than following complete mPFC lesion. In addition, the increase in perseverative responses observed in both NVH- and AVH-lesioned rats was lower than that obtained after entire mPFC or PRL-IL lesions. This is best explained by an inability for lesioned animals to withhold multiple inappropriate nose-poking behavior in an aperture in which a correct response had been emitted. Perseverative responses have already been observed in a delayed alternation task after inactivation of the $\mathrm{VH}$ (Maruki et al, 2001) and in a spontaneous alternation task, after VH damage (Stevens and Cowey, 1973). Interestingly, in acute and chronic schizophrenic patients, deficits in executive function associated with an increase in perseverative error scores have been demonstrated in the Wisconsin Card Sorting Test, a task sensitive to prefrontal cortical and also hippocampal dysfunction (Goldberg et al, 1987; Weinberger et al, 1992; Corcoran and Upton, 1993; Goldberg and Weinberger, 1994), and in a two-choice visual task with reinforcement (Lyon and Gerlach, 1988). Moreover, in the rat, bilateral knife-cut lesions of the perforant path also induced an increase of perseverative responses, implicating the entorhinal cortex/hippocampal circuitry in perseveration (Kirkby and Higgins, 1998). To summarize, given the connectivity between $\mathrm{VH}$ and the entorhinal cortex, in addition to the projections from the VH to mPFC (including PR and IL areas) (Swanson, 1981; Wyss, 1981; Jay and Witter, 1991; Gabbott et al, 2002), VH lesion-induced perturbations in these circuitries may result in perseverative responses and a reduction in accuracy as observed in $\mathrm{VH}$ lesioned rats upon baseline performance in the 5-CSRTT. These results also emphasize for the first time a role for the $\mathrm{VH}$ in visual attentional processes.

\section{Effects of Task Manipulation}

Challenges known to exacerbate deficits in attentional function were used (see Carli et al, 1983; Robbins et al, 1989; Jones et al, 1995). In summary, reducing the SD (a manipulation used to increase the attentional load) did not differentially affect the accuracy of VH-lesioned rats, and varying ITI (making the presentation of the stimuli temporally unpredictable) had the same effect in both sham and lesioned subjects. However, interpolating a distracting white noise immediately prior to the stimulus presentation induced a selective impairment in the ability of VH-lesioned rats to recognize the visual targets. Lesioned animals may thus be unable to ignore irrelevant or distracting stimuli, pointing to an impairment of selective attention function similar to that observed after mPFC lesions (Muir et al, 1996). Interestingly, NVH lesions have also been shown to induce a disruption of latent inhibition (Grecksch et al, 1999). This reflects a deficit in the ability to ignore irrelevant stimuli, a process depending on the integrity of the hippocampal formation (Gray et al, 1995). Acute schizophrenic patients also exhibit deficits in the ability to ignore irrelevant stimuli in a latent inhibition procedure (Baruch et al, 1988). There are also data to suggest that in chronic schizophrenic patients, latent inhibition deficits are present but masked by antipsychotic medications (Leumann et al, 2002). In addition, in stable chronic schizophrenics, higher levels of neuroleptics are associated with lessened distractibility in a reaction time task (Strauss et al, 1985), and an increased susceptibility to distraction while trying to process auditory information has been observed (Gold and Harvey, 1993).

Reducing the brightness level induced a selective impairment in the ability of $\mathrm{VH}$-lesioned rats to detect the visual targets. This sensitivity to reduced SI has been suggested by some to reflect visual dysfunction rather than attentional impairment (Robbins et al, 1989; Muir et al, 1992a,b) whereas for Jones et al (1995) sensorimotor deficits could not be responsible for these results. In our study, in good agreement with Jones et al (1995), deficits in visual function seem unlikely to be responsible for the observed effects since neonatal and adult $\mathrm{VH}$-lesioned rats did not exhibit visual impairment in the cued platform task of the Morris water maze paradigm (Le Pen et al, 2000). In good agreement with this hypothesis, it has also been shown in a latent inhibition paradigm that $\mathrm{NVH}$-lesioned rats acquired the conditioned reaction similarly to controls where conditioned stimuli were a combination of light and sound (Grecksch et al, 1999).

\section{Influence of Psychotomimetic Drugs upon Performance}

As previously reported (Jin et al, 1997), PCP increased correct latency, and both premature and perseverative responses, and also reduced choice accuracy. Interestingly, PCP exacerbated accuracy deficits in NVH- but not AVHlesioned rats. Other experiments have already shown an exacerbation of NVH lesion-induced abnormal behaviors in rats by PCP. These include hyper-responsiveness to PCPinduced locomotion (Hori et al, 2000; Kato et al, 2000) and PCP-induced retention deficits in a forced swim test used as a model of learning and memory (De Pablo et al, 1989; Hori et al, 2000).

Whether PCP-induced accuracy deficits are specific to attentional processes is an issue to be considered since systemically administered PCP has multiple effects on CNS function. Indeed, in our studies, and as already reported in the literature (Iwamoto, 1984; Hutson et al, 2000; Jacobs et al, 2000), PCP (3 mg/kg, s.c.) induced an increase of locomotion, stereotypies and ataxia and decreased rearing in Sprague-Dawley rats (data not shown). All these effects could contribute to the decrease in accuracy. However, this is unlikely since accuracy impairments induced by PCP $(3 \mathrm{mg} / \mathrm{kg}$, s.c.) in a 3-CSRTT were reversed by administration of a selective and high-affinity sigma antagonist that was inactive on PCP-induced hyperactivity in mice (Jin et al, 
1997) and on PCP-induced ataxia and rearing behaviour in rats (Takahashi et al, 2001). In addition, drug effects on accuracy have been shown to be dissociable from drug effects on locomotion or stereotypies. Indeed, nicotine increased accuracy of SD rats in the 5-CSRTT (Mirza and Bright, 2001) at a dose that also increased locomotion and stereotypies (Ksir, 1994). And Grottick and Higgins (2000) have shown that subchronic nicotine treatment increased accuracy in the 5-CSRTT whereas sensitization to nicotineinduced locomotor activity has been observed after the same treatment (Benwell and Balfour, 1992). Finally, in our studies, PCP $(3 \mathrm{mg} / \mathrm{kg}$, s.c. $)$ induced similar levels of locomotion and stereotypy in both control and NVHlesioned rats (data not shown) even when a hyperresponsiveness to PCP-induced attentional deficits was seen in NVH-lesioned rats.

The present results also replicate previous findings showing that systemic administration of d-amphetamine increases the speed of responding, premature and perseverative responses and also mildly decreases accuracy (Cole and Robbins, 1987; Harrison et al, 1997). However, we showed that, in contrast to PCP, amphetamine did not increase accuracy deficits seen in lesioned rats, whatever the age at lesion. Previous experiments performed in NVHlesioned rats have shown that cognitive impairments observed in the radial-arm maze were not exacerbated by amphetamine (Chambers et al, 1996) in contrast to hyperlocomotion (Lipska et al, 1993; Wan et al, 1996).

Thus, in good agreement with previous studies, we demonstrated that PCP, but not amphetamine, is able to exacerbate the cognitive deficits observed in NVH-lesioned rats.

These results obtained in NVH-lesioned rats can be compared with those obtained in schizophrenic patients, where PCP and ketamine (another NMDA receptor antagonist) have been reported to exacerbate both the positive and cognitive symptoms (Luby et al, 1959; Javitt and Zukin, 1991; Steinpreis, 1996; Malhotra et al, 1997; Jentsch and Roth, 1999), whereas amphetamine exacerbates positive symptoms (Angrist and Van Kammen, 1984; Lieberman et al, 1987; Wolkin et al, 1994) and can both improve or have no effect on negative symptoms (Goldberg et al, 1991; Sanfilipo et al, 1996). However, others have also reported an absence of ketamine effects on memory and cognition in schizophrenic patients (Laporte et al, 1996).

In conclusion, our study is the first that emphasizes an implication of the ventral hippocampus in visual attentional processes. In addition, our experiments suggest that $\mathrm{NVH}$ lesions in rats can simulate some aspects of the attentional and cognitive deficits observed in schizophrenic patients. This NVH lesion model of schizophrenia is also able to mimic the exacerbation of cognitive deficits seen in psychotic patients after PCP, but not amphetamine, challenge.

\section{REFERENCES}

Al-Amin HA, Weinberger DR, Lipska BK (2000). Exaggerated MK801 -induced motor hyperactivity in rats with the neonatal lesion of the ventral hippocampus. Behav Pharmacol 11: 269-278.

Al-Amin HA, Weinberger DR, Lipska BK (2001). Delayed onset of enhanced MK-801-induced motor hyperactivity after neonatal lesions of the rat ventral hippocampus. Biol Psychiatry 49: 528-539.

Angrist B, van Kammen DP (1984). CNS stimulants as a tool to study schizophrenia. Trends Neurosci 7: 388-390.

Baruch I, Hemsley DR, Gray JA (1988). Differential performance of acute and chronic schizophrenics in a latent inhibition task. J Nerv Ment Dis 176: 598-606.

Becker A, Grecksch G, Berstein H-G, Höllt V, Bogerts B (1999). Social behaviour in rats lesioned with ibotenic acid in the hippocampus: quantitative and qualitative analysis. Psychopharmacology 144: 333-338.

Benwell ME, Balfour DJ (1992). The effects of acute and repeated nicotine treatment on nucleus accumbens dopamine and locomotor activity. Br J Pharmacol 105: 849-856.

Black MD, Lister S, Hitchcock JM, Van Giersbergen P, Sorensen SM (1998). Neonatal hippocampal lesion model of schizophrenia in rats: sex differences and persistence of effects into maturity. Drug Dev Res 43: 206-213.

Brake WG, Sullivan RM, Flores G, Srivastava LK, Gratton A (1999). Neonatal ventral hippocampal lesions attenuate the nucleus accumbens dopamine response to stress: an electrochemical study in the adult rat. Brain Res 831: 25-32.

Breier A (1999). Cognitive deficit in schizophrenia and its neurochemical basis. Br J Psychiatry 37(Suppl): 16-18.

Carli M, Robbins TW, Evenden JL, Everitt BJ (1983). Effects of lesions to ascending noradrenergic neurones on performance of a 5-choice serial reaction time task in rats: implication theories of dorsal noradrenergic bundle function based on selective attention and arousal. Behav Brain Res 9: 361-380.

Carli M, Samanin R (1992). Serotonin2 receptor agonists and serotonergic anorectic drugs affect rats' performance differently in a five-choice serial reaction time task. Psychopharmacology 106: 228-234.

Chambers RA, Moore JM, McEvoy JP, Levin ED (1996). Cognitive effects of neonatal hippocampal lesions in rat model of schizophrenia. Neuropsychopharmacology 15: 587-594.

Cole BJ, Robbins TW (1987). Amphetamine impairs the discriminative performance of rats with dorsal noradrenergic bundle lesions on a 5-choice serial reaction time task: new evidence for central dopaminergic-noradrenergic interactions. Psychopharmacology 91: 458-466.

Corcoran R, Upton D (1993). A role for the hippocampus in card sorting? Cortex 29: 293-304.

Csernansky JG, Wang L, Jones D, Rastogi-Cruz D, Posener JA, Heydebrand G, Miller JP, Miller MI (2002). Hippocampal deformities in schizophrenia characterized by high dimensional brain mapping. Am J Psychiatry 159: 2000-2006.

De Pablo JM, Parra A, Segovia S, Guillanon A (1989). Learned immobility explains the behavior of rats in the forced swim test. Physiol Behav 46: 229-237.

Flores G, Barbeau D, Quirion R, Srivastava LK (1996). Decreased binding of dopamine D3 receptors in limbic subregions after neonatal bilateral lesion of rat hippocampus. J Neurosci 16: 2020-2026.

Gabbott P, Headlam A, Busby S (2002). Morphological evidence that CA1 hippocampal afferents monosynaptically innervate PVcontaining neurons and NADPH-diaphorase reactive cells in the medial prefrontal cortex (Areas 25/32) of the rat. Brain Res 946: 314-322.

Gold JM, Harvey PD (1993). Cognitive deficits in schizophrenia. Psychiatr ClinN Am 16: 295-312.

Goldberg TE, Bigelow LB, Weinberger DR, Daniel DG, Kleinman JE (1991). Cognitive and behavioral effects of the coadministration of dextroamphetamine and haloperidol in schizophrenia. Am J Psychiatry 148: 78-84.

Goldberg TE, Weinberger DR (1994). Schizophrenia, training paradigms, and the Wisconsin Card Sorting Test redux. Schizophr Res 11: 291-296. 
Goldberg TE, Weinberger DR, Berman KF, Pliskin NH, Podd MH (1987). Further evidence for dementia of the prefrontal type in schizophrenia? A controlled study of teaching the Wisconsin Card Sorting Test. Arch Gen Psychiatry 44: 1008-1014.

Gomez-Serrano M, Tonelli L, Listwak S, Sternberg E, Riley AL (2001). Effects of cross fostering on open-field behavior, acoustic startle, lipopolysaccharide-induced corticosterone release, and body weight in Lewis and Fischer rats. Behav Genet 31: 427-436.

Gray JA, Joseph MH, Hemsley DR, Young AMJ, Warburton EC, Boulenguez $\mathrm{P}$ et al (1995). The role of mesolimbic dopaminergic and retrohippocampal afferents to the nucleus accumbens in latent inhibition: implications for schizophrenia. Behav Brain Res 71: 19-31.

Grecksch G, Bernstein HG, Becker A, Höllt V, Begerts B (1999). Disruption of latent inhibition in rats with postnatal hippocampal lesions. Neuropsychopharmacology 20: 525-532.

Grottick AJ, Higgins GA (2000). Effect of subtype selective nicotinic compounds on attention as assessed by the fivechoice serial reaction time task. Behav Brain Res 117: 197-208.

Harrison AA, Everitt BJ, Robbins TW (1997). Doubly dissociable effects of median- and dorsal-raphé lesions on the performance of the five-choice serial reaction time test of attention in rats. Behav Brain Res 89: 135-149.

Hock BJ, Bunsey MD (1998). Differential effects of dorsal and ventral hippocampal lesions. J Neurosci 18: 7027-7032.

Hori T, Subramaniam S, Srivastava LK, Quirion R (2000). Behavioral and neurochemical alterations following repeated phencyclidine administration in rats with neonatal ventral hippocampal lesions. Neuropharmacology 39: 2478-2491.

Hughes C, Kumari V, Soni W, Das M, Binneman B, Drozd S et al (2003). Longitudinal study of symptoms and cognitive function in chronic schizophrenia. Schizophr Res 59: 137-146.

Hutson PH, Barton CL, Jay M, Blurton P, Burkamp F, Clarkson R et al (2000). Activation of mesolimbic dopamine function by phencyclidine is enhanced by $5-\mathrm{HT}(2 \mathrm{C} / 2 \mathrm{~B})$ receptor antagonists: neurochemical and behavioural studies. Neuropharmacology 39: 2318-2328.

Iwamoto ET (1984). An assessment of the spontaneous activity of rats administered morphine, phencyclidine, or nicotine using automated and observational methods. Psychopharmacology 84: 374-382.

Jacobs PS, Taylor BM, Bardgett ME (2000). Maturation of locomotor and Fos responses to the NMDA antagonists, PCP and MK-801. Dev Brain Res 122: 91-95.

Javitt DC, Zukin SR (1991). Recent advances in the phencyclidine model of schizophrenia. Am J Psychiatry 148: 1301-1308.

Jay TM, Witter MP (1991). Distribution of hippocampal CA1 and subicular efferents in the prefrontal cortex of the rat studied by means of anterograde transport of Phaseolus vulgaris-leucoagglutinin. J Comp Neurol 313: 574-586.

Jentsch JD, Roth RH (1999). The neuropsychopharmacology of phencyclidine: from NMDA receptor hypofunction to the dopamine hypothesis of schizophrenia. Neuropsychopharmacology 20: 201-225.

Jin J, Yamamoto T, Watanabe S (1997). The involvement of sigma receptors in the choice reaction performance deficits induced by phencyclidine. Eur J Pharmacol 319: 147-152.

Jones DNC, Barnes JC, Kirkby DL, Higgins GA (1995). Ageassociated impairments in a test of attention: evidence for involvement of cholinergic systems. J Neurosci 15: 7282-7292.

Joober R, Rouleau GA, Lal S, Dixon M, O'Driscoll G, Palmour R et al (2002). Neuropsychological impairments in neurolepticresponder $v s$-nonresponder schizophrenic patients and healthy volunteers. Schizophr Res 53: 229-238.

Kato K, Shishido T, Ono M, Shishido K, Kobayashi M, Suzuki H et al (2000). Effects of phencyclidine on behavior and extracellular levels of dopamine and its metabolites in neonatal ventral hippocampal damaged rats. Psychopharmacology 150: 163-169.

Kirkby DL, Higgins GA (1998). Characterization of perforant path lesions in rodent models of memory and attention. Eur $J$ Neurosci 10: 823-838.

Ksir C (1994). Acute and chronic nicotine effects on measures of activity in rats: a multivariate analysis. Psychopharmacology 115: 105-109.

LaPorte DJ, Lahti AC, Koffel B, Tamminga CA (1996). Absence of ketamine effects on memory and other cognitive functions in schizophrenia patients. $J$ Psychiatr Res 30: 321-330.

Leonard JA (1959). Five-choice serial reaction apparatus. Report 326. Cambridge, MA: Medical Research Council. Appl Psychol Unit Rep.

Leonard JA (1961). Choice reaction time experiments and information theory. In: Cherry C (ed) Information Theory. Butterworths: London.

Le Pen G, Grottick AJ, Higgins GA, Martin JR, Jenck F, Moreau J-L (2000). Spatial and associative learning deficits induced by neonatal excitotoxic hippocampal damage in rats: further evaluation of an animal model of schizophrenia. Behav Pharmacol 11: 257-268.

Le Pen G, Gaudet L, Mortas P, Mory R, Moreau JL (2002). Deficits in reward sensitivity in a neurodevelopmental rat model of schizophrenia. Psychopharmacology 161: 434-441.

Leumann L, Feldon J, Vollenweider FX, Ludewig K (2002). Effects of typical and atypical antipsychotics on prepulse inhibition and latent inhibition in chronic schizophrenia. Biol Psychiatry 52: 729-739.

Lieberman JA, Kane JM, Alvir J (1987). Provocative tests with psychostimulant drugs in schizophrenia. Psychopharmacology 91: 415-433.

Lipska BK, Aultman JM, Verma A, Weinberger DR, Moghaddam B (2002). Neonatal damage of the ventral hippocampus impairs working memory in the rat. Neuropsychopharmacology 27: 47-54.

Lipska BK, Chrapusta SJ, Egan MF, Weinberger DR (1995a). Neonatal excitotoxic ventral hippocampal damage alters dopamine response to mild repeated stress and to chronic haloperidol. Synapse 20: 125-130.

Lipska BK, Jaskiw GE, Weinberger DR (1993). Postpubertal emergence of hyperresponsiveness to stress and to amphetamine after neonatal excitotoxic hippocampal damage: a potential animal model of schizophrenia. Neuropsychopharmacology 9: 67-75.

Lipska BK, Swerdlow NR, Geyer MA, Jaskiw GE, Braff DL, Weinberger DR (1995b). Neonatal excitotoxic hippocampal damage in rats causes post-pubertal changes in prepulse inhibition of startle and its disruption by apomorphine. Psychopharmacology 122: 35-43.

Lipska BK, Weinberger DR (1993). Delayed effects of neonatal hippocampal damage on haloperidol-induced stereotypic behaviors in the rat. Dev Brain Res 75: 213-222.

Liu D, Diorio J, Day JC, Francis DD, Meaney MJ (2000). Maternal care, hippocampal synaptogenesis and cognitive development in rats. Nat Neurosci 3: 799-806.

Luby E, Cohen B, Rosenbaum G, Gottlieb J, Kelley R (1959). Study of a new schizophrenomimetic drug-Sernyl. Arch Neurol Psychiatry 81: 363-369.

Lyon N, Gerlach J (1988). Perseverative structuring of responses by schizophrenic and affective disorder patients. J Psychiatr Res 22: 261-277.

Malhotra AK, Pinals DA, Adler CM, Elman I, Clifton A, Pickar D et al (1997). Ketamine-induced exacerbation of psychotic symptoms and cognitive impairment in neuroleptic-free schizophrenics. Neuropsychopharmacology 17: 141-150. 
Maruki K, Izaki Y, Hori K, Nomura M, Yamauchi T (2001). Effects of rat ventral and dorsal hippocampus temporal inactivation on delayed alternation task. Brain Res 895: 273-276.

Mirsky AF, Rosvold HE (1960). The use of psychoactive drugs as a neuropsychological tool in studies of attention in man. In: Uhr I, Miller JG (eds) Drugs and Behavior. Wiley: New York. pp 375-392.

Mirza NR, Bright JL (2001). Nicotine-induced enhancements in the five-choice serial reaction time task in rats are strain-dependent. Psychopharmacology 154: 8-12.

Moser MB, Moser EI, Forrest E, Andersen P, Morris RG (1995). Spatial learning with a minislab in the dorsal hippocampus. Proc Natl Acad Sci USA 92: 9697-9701.

Muir JL, Dunnett SB, Robbins TW, Everitt BJ (1992a). Attentional functions of the forebrain cholinergic systems: effects of intraventricular hemicholinium, physostigmine, basal forebrain lesions and intracortical grafts on a multiple-choice serial reaction time task. Exp Brain Res 89: 611-622.

Muir JL, Everitt BJ, Robbins TW (1994). AMPA-induced excitotoxic lesions of the basal forebrain: a significant rôle for the cortical cholinergic system in attentional function. J Neurosci 14: 2313-2326.

Muir JL, Everitt BJ, Everitt TW (1996). The cerebral cortex of the rat and visual attentional function: dissociable effects of mediofrontal, cingulate, anterior dorsolateral, and parietal cortex lesions on a five-choice serial reaction time task. Cereb Cortex 6: $470-481$.

Muir JL, Robbins TW, Everitt BJ (1992b). Disruptive effects of muscimol infused into the basal forebrain on conditional discrimination and visual attention: differential interactions with cholinergic mechanisms. Psychopharmacology 107: 541550.

Passetti F, Chudasama Y, Robbins TW (2002). The frontal cortex of the rat and visual attentional performance: dissociable functions of distinct medial prefrontal subregions. Cereb Cortex 12: 12541268.

Robbins TW, Everitt BJ (1995). Arousal systems and attention. In: Gazziniga MS (ed) The Cognitive Neurosciences. MIT Press: Cambridge, MA. pp 703-720.

Robbins TW, Everitt BJ, Marston HM, Wilkinson J, Jones GH, Page KJ (1989). Comparative effects of ibotenic acid- and quisqualic acid-induced lesions of the substantia innominata on attentional function in the rat: further implications for the role of the cholinergic neurons of the nucleus basalis in cognitive processes. Behav Brain Res 35: 221-240.

Sams-Dodd F, Lipska BK, Weinberger DR (1997). Neonatal lesions of the rat ventral hippocampus result in hyperlocomotion and deficits in social behaviour in adulthood. Psychopharmacology 132: 303-310.

Sanfilipo M, Wolkin A, Angrist B, van Kammen DP, Duncan E, Wieland S, Cooper TB, Peselow ED, Rotrosen J (1996). Amphetamine and negative symptoms of schizophrenia. Psychopharmacology 123: 211-214.

Sarter M, Bruno JP (2000). Cortical cholinergic inputs mediating arousal, attentional processing and dreaming: differential afferent regulation of the basal forebrain by telencephalic and brainstem afferents. Neuroscience 95: 933-952.

Sharma T (1999). Cognitive effects of conventional and atypical antipsychotics in schizophrenia. $B r J$ Psychiatry 38(Suppl): $44-51$.

Steinpreis RE (1996). The behavioral and neurochemical effects of phencyclidine in humans and animals: some implications for modeling psychosis. Behav Brain Res 74: 45-55.

Stevens R, Cowey A (1973). Effects of dorsal and ventral hippocampal lesions on spontaneous alternation, learned alternation and probability learning in rats. Brain Res 52: 203-224.

Strauss ME, Lew MF, Coyle JT, Tune LE (1985). Psychopharmacologic and clinical correlates of attention in chronic schizophrenia. Am J Psychiatry 142: 497-499.

Stubley-Weatherly LA, Harding JW, Wright JW (1996). Effects of kainic acid-induced hippocampal lesions on spatial and contextual learning and memory in rats. Brain Res 716: 29-38.

Swanson LW (1981). A direct projection from Ammon's horn to prefrontal cortex in the rat. Brain Res 217: 150-154.

Takahashi S, Takagi K, Horikomi K (2001). Effects of a novel, selective, sigma1-ligand, MS-377, on phencyclidineinduced behaviour. Naunyn Schmiedebergs Arch Pharmacol 364: $81-86$.

Wan RQ, Giovanni A, Kafka SH, Corbett R (1996). Neonatal hippocampal lesions induced hyperresponsiveness to amphetamine: behavioral and in vivo microdialysis studies. Behav Brain Res 78: 211-223.

Weinberger DR (1986). The pathogenesis of schizophrenia: a neurodevelopmental theory. In: Nasrallah HA, Weinberger DR (eds) The Neurology of Schizophrenia. Elsevier: Holland. pp 397406.

Weinberger DR (1999). Cell biology of the hippocampal formation in schizophrenia. Biol Psychiatry 45: 385-394.

Weinberger DR, Berman KF, Suddath R, Torrey EF (1992). Evidence of dysfunction of a prefrontal-limbic network in schizophrenia: a magnetic resonance imaging and regional cerebral blood flow study of discordant monozygotic twins. Am J Psychiatry 149: 890-897.

Weinberger DR, Galhofer B (1997). Cognitive function in schizophrenia. Int Clin Psychopharmacol 12(Suppl 4): S39-S36.

Wolkin A, Sanfilipo M, Angrist B, Duncan E, Wieland S, Wolf AP et al (1994). Acute d-amphetamine challenge in schizophrenia: effects on cerebral glucose utilization and clinical symptomatology. Biol Psychiatry 36: 317-325.

Wood GK, Marcotte ER, Quirion R, Srivastava LK (2001). Strain differences in the behavioural outcome of neonatal ventral hippocampal lesions are determined by the postnatal environment and not genetic factors. Eur J Neurosci 14: 1030-1034.

Wyss JM (1981). An autoradiographic study of the efferent connections of the entorhinal cortex in the rat. J Comp Neurol 199: 495-512.

Zaidel DW, Esiri MM, Harrison PJ (1997). Size, shape, and orientation of neurons in the left and right hippocampus: investigation of normal asymmetries and alterations in schizophrenia. Am J Psychiatry 154: 812-818. 